\title{
Star-forming QSO host galaxies
}

\author{
P. D. Barthel
}

\begin{abstract}
Kapteyn Astronomical Institute, University of Groningen, PO Box 800, 9700 AV Groningen, The Netherlands e-mail: pdb@astro.rug.nl
\end{abstract}

Received 8 June 2005 / Accepted 26 July 2006

\begin{abstract}
Context. The recent finding of substantial masses of cold molecular gas as well as young stellar populations in the host galaxies of intermediate luminosity quasars is at odds with results of Hubble Space Telescope imaging studies since the latter appear to yield mature, quiescent early type hosts.

Aims. The characterization as "quiescent" for the host galaxies of QSOs is being addressed.

Methods. The radio and far-infrared properties of both the HST sample and a larger comparison sample of uv-excess selected radioquiet QSOs are being analyzed.

Results. Consistency is found with substantial recent or ongoing star-formation activity.
\end{abstract}

Key words. quasars: general - galaxies: starburst

\section{Introduction}

Quasi-stellar objects, or QSOs, represent episodes of extreme nuclear luminosity in galaxies, over a wide wavelength range. QSOs display a considerable dispersion in range-loudness, which is commonly expressed (Kellermann et al. 1989) as ratio $R$ of cm-radio over blue (4400 $\AA$ ) flux density. Radio-quiet QSOs typically have $R \lesssim 1$, while radio-loud QSOs are characterized with $R$ in excess of 10. QSO host galaxies - both of the radio-loud and the radio-quiet subgroup - have been targets of studies for many years. Early investigations (e.g., Hutchings et al. 1984) revealed luminous hosts, frequently with close companions, and sometimes displaying spectroscopic features of young stars. Systematic studies of QSO host galaxies became possible with the refurbished Hubble Space Telescope (HST): host galaxies of different nature were identified (Bahcall et al. 1997). Using bulge-black hole mass correlations, these studies permitted assessment of the QSO accretion rates, and rates up to 20 per cent of the Eddington values were inferred (McLeod et al. 1999). Ground-based near-infrared studies of samples of both radio-loud and radio-quiet hosts as well as powerful $\left(P_{\text {radio }}>\right.$ $10^{27} \mathrm{~W} / \mathrm{Hz}$ ) radio galaxy hosts indicated (Taylor et al. 1996) the predominance of very luminous spheroidal hosts, particularly in the two radio-loud AGN populations. These results were confirmed by imaging studies from space, using HST. In the final paper (Dunlop et al. 2003) in a series dealing with WFPC2 studies of the host galaxies of matched samples of radio-loud and radio-quiet QSOs as well as radio galaxies, it was concluded from 2D model fitting procedures that "... for nuclear luminosities brighter than $V=23.5$ the hosts are virtually all massive ellipticals with properties indistinguishable from those of quiescent, evolved low-redshift ellipticals of comparable mass".

Powerful radio-galaxies are long known to be hosted by massive ellipticals, which are however sometimes of disturbed morphology (e.g., Heckman et al. 1986). While the HST imaging results (Dunlop et al. 2003) seem consistent with AGN unification models (involving obscuring dusty circumnuclear tori - e.g., Barthel 1989; Urry \& Padovani 1993) it must be stressed that also the presence of extended dust, cold gas, and young stars in many radio-loud AGN has been reported (Hes et al. 1995; Martel et al. 1999; Tadhunter et al. 2005; Van Gorkom et al. 1989), which in turn is difficult to reconcile with the picture of quiescent ellipticals. Similar contrasting views exist on the hosts of radio-quiet QSOs, i.e., QSOs displaying radio-optical flux ratio's of order unity or smaller. Whereas - as mentioned above - 2D model fitting of the red starlight distributions generally seems to yield dominant spheroids, the properties of the interstellar media of several radio-quiet QSO hosts do not support the quiescent massive elliptical classification. The recent finding (Evans et al. 2001; Scoville et al. 2003) of substantial masses of cold molecular gas, as well as several cases (Canalizo \& Stockton 2001) of young circumnuclear stars in radio-quiet QSO host galaxies is at odds with conclusions of the imaging studies. Some radio-quiet QSOs must be (Clements 2000) post-starburst systems, of which QSO UN J1025-440 provides a spectacular example (Brotherton et al. 1999). An extensive radio-optical study (Miller et al. 1993) using 89 low redshift QSOs, indicated that roughly half of the weak radio emission in the radio-quiet subgroup might draw from host star-formation. A study of the integrated radio and farinfrared emission in various classes of active objects (Colina \& Perez-Olea 1995) confirmed that suspicion. The HST results (Dunlop et al. 2003) are also inconsistent with modeling studies (Rowan-Robinson 1995) of infrared spectral energy distributions (SEDs), concluding “... an AGN is inevitably accompanied by a star-burst ..."

We therefore examine in detail radio imaging and farinfrared photometric data for the radio-quiet subset of the HST QSO sample of Dunlop et al. (2003), and demonstrate from comparison with similar data for the uv-excess selected PG QSOs that the radio and far-infrared properties of both samples are in fact consistent with the presence of gas-rich star-forming 
interstellar media in the AGN hosts. So while we acknowledge the presence of a massive spheroid as a necessary condition for the nuclear activity to occur, we conclude that the circumnuclear regions in the host galaxies of intermediate luminosity QSOs must contain young stars, supernova remnants, and starburst heated dust. That conclusion is also in line with results of recent SDSS studies (Kaufmann et al. 2003), suggesting that many AGN are post-starburst systems, and recent ISO studies (Haas et al. 2003).

\section{Sample and analysis}

The HST/Dunlop et al. radio-quiet (i.e., radio-weak, not radiosilent) QSO sample (Dunlop et al. 2003) consists of thirteen objects, in the redshift range $0.1<z<0.25$. Of these thirteen, QSO $1635+119$ is associated with an extended ( $\sim 1$ arcmin) radio source of luminosity $P(5 \mathrm{GHz})=5 \times 10^{24} \mathrm{~W} / \mathrm{Hz}$; combining this with its $R(5 \mathrm{GHz})$ value of 67 , we contend that this object should be formally classified as a radio-loud AGN (its optical magnitude is too faint for a QSO). Note that radio-emission in radioquiet QSOs is generally weak (in absolute and relative terms), and confined to the nuclear and/or circumnuclear regions of the QSO host galaxy. The thirteen HST QSOs will be compared with Palomar-Green (PG - Schmidt \& Green 1983) QSOs in the same redshift range, $0.1<z<0.25$. The latter is a well known sample of QSOs selected through their ultraviolet-excess, and has welldocumented properties (Sanders et al. 1989): thirty-one radioquiet PG QSOs are found in the relevant redshift range. Both samples are tabulated in the table (note: six QSOs in common). Besides name, redshift, and absolute optical magnitude, we list flux densities at $60 \mu \mathrm{m}$ and $5 \mathrm{GHz}$, inferred luminosities at $60 \mu \mathrm{m}$ and $5 \mathrm{GHz}$, radio core fractions $f_{\mathrm{c}}$ at $5 \mathrm{GHz}, u$-parameters (logarithmic ratio's of the $60 \mu \mathrm{m}$ and $5 \mathrm{GHz}$ flux densities), for the integrated $(u)$ and the extended, radio-core subtracted $\left(u^{\prime}\right)$ emission, and relevant references. Core fractions $f_{\mathrm{c}}$ were obtained by comparing the $5 \mathrm{GHz}$ flux densities obtained at low resolution (typically 18 arcsec - Kellermann et al. 1989) with those at high resolution ( $\$ 0.5 \mathrm{arcsec})$. In several cases IRAS ADDSCAN routines were used to re-assess the $60 \mu \mathrm{m}$ properties, yielding new detections as well as improved upper limits. Having $-25 \lesssim M_{\text {abs }} \lesssim-23$, the objects comprise a representative sample of intermediate luminosity radio-quiet QSOs.

\section{Results}

The $60 \mu \mathrm{m}$ far-infrared luminosities, for the HST and the PG samples, run from 10.5 to 12.5 (in $\log L_{\odot}$ ). The former has five upper limits, the latter eight. The radio luminosities run from $10^{22}$ to $10^{24} \mathrm{~W} / \mathrm{Hz}$, with one $10^{24.7}$ in the Dunlop set (the radio-loud object). Although the fractions of $60 \mu \mathrm{m}$ nondetections (5/13 vs. 8/31) suggest otherwise, survival analysis (taking the upper limits into account) cannot exclude that the two samples were drawn from one and the same parent population. More sensitive infrared photometry is needed to re-address that issue. The FIR and radio luminosities however, indicate that the hosts cannot be quiescent early type galaxies. The Dunlop and PG QSO sample display $60 \mu \mathrm{m}$ luminosities which are orders of magnitude in excess of (IRAS/ISO) luminosities for massive inactive ellipticals, which are generally (Walsh et al. 1989) in the range $10^{7}-10^{9} L_{\odot}$. There would be an obvious explanation for this fact, if all far-infrared and radio emission drew from the nuclear activity, but this appears not to be the case as we will demonstrate in the following.

Figure 1 compares the optical nuclear luminosities and the $60 \mu \mathrm{m}$ luminosities, for the QSOs in both samples. While the two

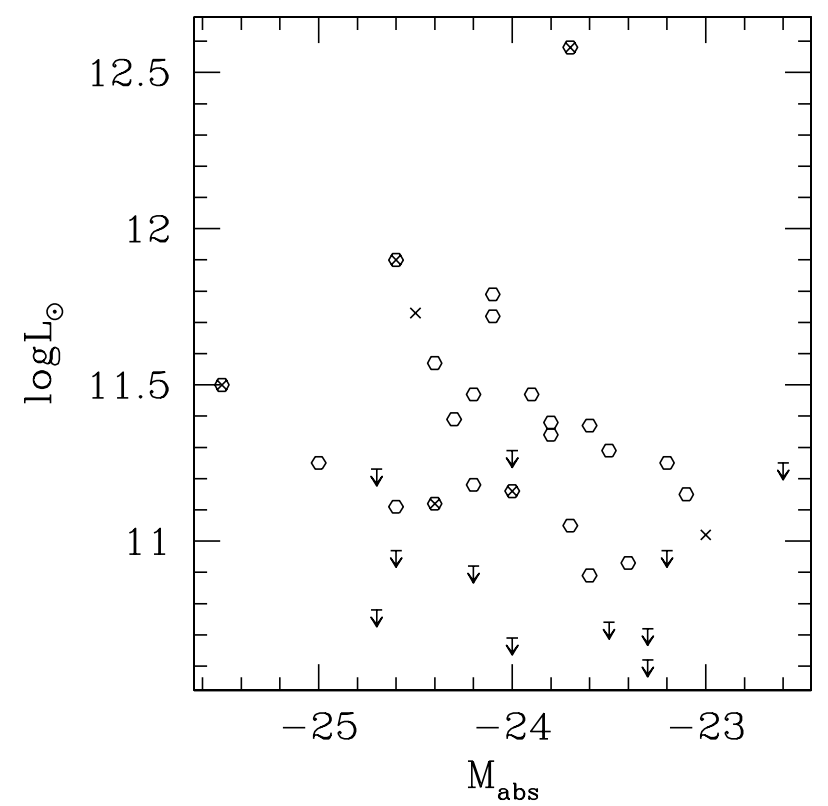

Fig. 1. $60 \mu \mathrm{m}$ FIR luminosities (expressed in $\log L_{\odot}$ ) versus absolute (nuclear) optical magnitudes, for the QSOs from both samples (with the exception of $0257+024,1635+119$ and $2344+184$, whose optical faintness formally disqualifies these object as QSOs). The crosses mark QSOs from the HST sample, the open hexagons mark QSOs from the PG-sample. For the sake of simplicity, the $R$-values for the HST sample are combined with the $B$-values for the PG-sample ( $R$-values used for the common objects), since the (small) color transformations are uncertain due to intrinsic spread in the optical continuum slopes and varying emission line contributions.

quantities are broadly correlated, a substantial scatter is seen. Figure 1 - dealing with a small sample in a fairly narrow redshift range - confirms results from the Haas et al. (2003) study of a large sample of PG QSOs studied with ISO. This study reports the presence of high infrared luminosities implying large dust masses ranging from $10^{6}$ to $10^{8.5} M_{\odot}$. However, in contrast to the near-IR $(3-10 \mu \mathrm{m})$ and the mid-IR $(10-40 \mu \mathrm{m})$ luminosity, being fairly well correlated with the QSO (blue) luminosity, the far-IR emission $(40-150 \mu \mathrm{m})$ is found to display a scatter of about a factor 10 in its correlation with that QSO luminosity. This suggests that FIR-emission surely draws from the active galactic nucleus but must in addition have a significant contribution from stellar heating. As Haas et al. (2003) stress, the large amounts of dust in QSOs would require that the QSO phase was at least preceeded by a starburst. We investigate this further by addressing the radio continuum emission as this offers an unobscured probe of host galaxy star-formation.

As demonstrated earlier (e.g., Wilson 1988), radio-emission in active galaxies can be separated into two components, one deriving from the nuclear activity and one from host galaxy starformation. Subtracting the AGN-driven component from the integrated radio emission, an improved correlation with respect to the integrated long wavelength far-infrared emission (which draws from star-formation) is being found. The u-parameter, as defined above, is generally used for this purpose. This parameter relates the $60 \mu \mathrm{m}$ FIR emission to the $20 \mathrm{~cm}$ or $6 \mathrm{~cm}$ radio emission. Star-forming objects are characterized (Condon \& Broderick 1988) with average $(20 \mathrm{~cm})$ parameter $u_{20}=2.0$, and $\pm 2 \sigma$-range $1.6-2.4$, i.e., $1.4 \mathrm{GHz}$ radio emission with strength between 0.4 and 2.5 per cent of the $60 \mu \mathrm{m}$ FIR emission ( 1 per cent in the mean). Using the standard radio spectral 
Table 1. The table lists integrated flux densities, in $\mathrm{mJy}$, at $60 \mu \mathrm{m}$ FIR and $5 \mathrm{GHz}(6 \mathrm{~cm})$ radio, radio core fractions $f_{\mathrm{c}}$ (fraction of $5 \mathrm{GHz}$ radio emission in subarcsec core), luminosities at $60 \mu \mathrm{m}$ (in solar units) and spectral power at $5 \mathrm{GHz}\left(\mathrm{W} / \mathrm{Hz}\right.$ ). Furthermore, $u_{6}$-parameters, addressing conformity to the FIR-radio correlation for the integrated $(u)$ as well as the core-subtracted emission $\left(u^{\prime}\right)$. Parameter $u_{6}$ is defined as $\log S(60 \mu \mathrm{m}) / S(5 \mathrm{GHz})$, whereas $u_{6}^{\prime}=\log S(60 \mu \mathrm{m}) / S\left(5 \mathrm{GHz}\right.$, extended); star-formation generally yields $u_{6}$-parameters in the range $2.0-2.8$; radio-loud AGN (with strong non-thermal core and lobe emission) have $u_{6} \ll 2$, incl. negative values. Values for the Hubble parameter and deceleration parameter of 50 and 0.5 respectively, in a $\Lambda=0$ universe have been used to compute source intrinsic quantities. The 13 QSOs from the HST sample (Dunlop et al. 2003) are followed by the 31 PG (Schmidt \& Green 1983) QSOs: the former have absolute (nuclear) $R$-band magnitudes listed, the latter $B$-band, or $V$-band (PG 0906+484). References: BWE91 - Becker et al. (1991), D03 - Dunlop et al. (2003), K89 - Kellermann et al. (1989), Ku98 - Kukula et al. (1998), S89 - Sanders et al. (1989), T96 - Taylor et al. (1996).

\begin{tabular}{|c|c|c|c|c|c|c|c|c|c|c|c|}
\hline QSO & $z$ & $M_{\mathrm{abs}}$ & $S(60 \mu)$ & $S(5 \mathrm{GHz})$ & $\log L(60)$ & $\log P(5)$ & $f_{\mathrm{c}}$ & $u_{6}$ & $u_{6}^{\prime}$ & Ref.(60 $\mu)$ & Ref.(5 GHz) \\
\hline PG0052+251 & 0.155 & -24.4 & 93 & 0.74 & 11.12 & 22.88 & 0.57 & 2.10 & 2.47 & S89 & K89 \\
\hline $0054+144$ & 0.171 & -24.5 & 307 & $1.5^{a}$ & 11.73 & 23.28 & 0.5 & 2.31 & 2.61 & $\mathrm{FSC}^{b}$ & $\mathrm{NVSS}^{c}, \mathrm{Ku} 98$ \\
\hline PG $0157+001$ & 0.164 & -23.7 & 2377 & 8.0 & 12.58 & 23.97 & $0.4^{d}$ & 2.47 & 2.69 & S89 & K89 \\
\hline 0204+292 & 0.109 & -23.0 & 150 & $\lesssim 10$ & 11.02 & $\lesssim 23.7$ & & $\gtrsim 1.2$ & & ADDSCAN $^{e}$ & T96 \\
\hline $0244+194$ & 0.176 & -23.2 & $<50$ & 0.18 & $<10.97$ & 22.40 & & $<2.4$ & & ADDSCAN & D03 \\
\hline $0257+024$ & 0.115 & -19.7 & $<50$ & 3.4 & $<10.59$ & 23.29 & & $<1.2$ & & ADDSCAN & $\mathrm{Ku} 98$ \\
\hline PG 0923+201 & 0.190 & -24.6 & 361 & 0.25 & 11.90 & 22.59 & 0.6 & 3.2 & 3.5 & FSC & K89, D03 \\
\hline PG 0953+414 & 0.239 & -25.5 & 90 & 1.9 & 11.50 & 23.67 & 0.1 & 1.68 & 1.75 & ADDSCAN & K89, D03 \\
\hline PG $1012+008$ & 0.185 & -24.0 & 70 & 1.0 & 11.16 & 23.17 & 0.74 & 1.85 & 2.43 & ADDSCAN & K89 \\
\hline $1549+203$ & 0.250 & -24.0 & $<50$ & $<0.12$ & $<11.29$ & $<22.5$ & & & & ADDSCAN & D03 \\
\hline $1635+119$ & 0.146 & -21.5 & $<50$ & 49 & $<10.80$ & 24.65 & RL & $<0$ & & ADDSCAN & BWE91 \\
\hline $2215-037$ & 0.241 & -22.6 & $<50$ & 0.13 & $<11.25$ & 22.50 & & $<2.6$ & & ADDSCAN & D03 \\
\hline $2344+184$ & 0.138 & -20.3 & 100 & 0.19 & 11.05 & 22.20 & & 2.72 & & ADDSCAN & D03 \\
\hline PG $0026+129$ & 0.142 & -24.7 & $<50$ & 5.1 & $<10.78$ & 23.65 & 0.04 & $<1.0$ & $<2.4$ & ADDSCAN & K89 \\
\hline PG $0052+251$ & 0.155 & -24.4 & 93 & 0.74 & 11.12 & 22.88 & 0.57 & 2.10 & 2.47 & S89 & K89 \\
\hline PG $0157+001$ & 0.164 & -24.8 & 2377 & 8.0 & 12.58 & 23.97 & $0.4^{d}$ & 2.47 & 2.69 & S89 & K89 \\
\hline PG 0804+761 & 0.100 & -23.7 & 191 & 2.38 & 11.05 & 23.00 & 0.41 & 1.83 & 2.13 & S89 & K89 \\
\hline PG $0838+770$ & 0.131 & -23.2 & 174 & $<0.4$ & 11.25 & $<22.5$ & & $>2.6$ & & S89 & $\mathrm{NED}^{f}, \mathrm{~K} 89$ \\
\hline PG 0906+484 & 0.118 & -23.1 & 172 & $<0.5$ & 11.15 & $<22.5$ & & $>2.5$ & & S89 & NED \\
\hline PG 0923+201 & 0.190 & -24.2 & 361 & 0.25 & 11.90 & 22.59 & 0.6 & 3.2 & 3.5 & FSC & K89, D03 \\
\hline PG 0947+396 & 0.206 & -24.1 & 201 & 0.31 & 11.72 & 22.75 & 0.77 & 2.81 & 3.45 & S89 & K89 \\
\hline PG $0953+414$ & 0.239 & -25.7 & 90 & 1.9 & 11.50 & 23.67 & 0.1 & 1.68 & 1.75 & ADDSCAN & K89, D03 \\
\hline PG 1001+054 & 0.161 & -23.8 & 140 & 0.80 & 11.34 & 22.95 & 0.3 & 2.24 & 2.40 & NED & K89 \\
\hline PG $1012+008$ & 0.185 & -24.3 & 70 & 1.00 & 11.16 & 23.17 & 0.74 & 1.85 & 2.43 & ADDSCAN & K89 \\
\hline PG $1048+342$ & 0.167 & -24.2 & $<50$ & 0.01 & $<10.92$ & 22.40 & & $<3.7$ & & ADDSCAN & K89 \\
\hline PG $1114+445$ & 0.144 & -23.6 & 191 & 0.22 & 11.37 & 22.28 & 0.91 & 2.94 & 3.98 & S89 & K89 \\
\hline PG $1115+407$ & 0.154 & -23.8 & 170 & 0.30 & 11.38 & 22.4 & 0.8 & 2.75 & 3.45 & ADDSCAN & K89 \\
\hline PG $1121+422$ & 0.234 & -24.7 & $<50$ & $<0.1$ & $<11.23$ & $<22.4$ & & & & ADDSCAN & K89 \\
\hline PG $1151+117$ & 0.176 & -24.6 & $<50$ & 0 & $<10.97$ & $<21.1$ & & & & ADDSCAN & K89 \\
\hline PG $1202+281$ & 0.165 & -25.0 & 110 & 0.83 & 11.25 & 22.9 & 0.78 & 2.12 & 2.78 & S89 & K89 \\
\hline PG $1307+085$ & 0.155 & -24.6 & 90 & 0.35 & 11.11 & 22.64 & & 2.41 & & ADDSCAN & K89 \\
\hline PG $1322+659$ & 0.168 & -24.2 & 90 & 0.20 & 11.18 & 22.38 & & 2.65 & & ADDSCAN & K89 \\
\hline PG $1352+183$ & 0.158 & -24.2 & 197 & 0.25 & 11.47 & 22.43 & & 2.90 & & NED & K89 \\
\hline PG $1402+261$ & 0.164 & -24.4 & 229 & 0.62 & 11.57 & 22.8 & 0.4 & 2.57 & 2.79 & S89 & K89 \\
\hline PG $1415+451$ & 0.114 & -23.4 & 112 & 0.40 & 10.93 & 23.3 & 0.6 & 2.45 & 2.85 & S89 & K89 \\
\hline PG 1416-129 & 0.129 & -24.0 & $<50$ & 3.60 & $<10.69$ & 23.4 & 0.22 & $<1.14$ & $<1.25$ & ADDSCAN & K89 \\
\hline PG $1427+480$ & 0.221 & -24.3 & 82 & 0.02 & 11.39 & 21.6 & & 3.6 & & ADDSCAN & K89 \\
\hline PG 1435-067 & 0.129 & -23.9 & 304 & 0.19 & 11.47 & 22.14 & 1 & 3.20 & 3.20 & NED & K89 \\
\hline PG $1519+226$ & 0.137 & -23.5 & $<50$ & 1.50 & $<10.74$ & 23.08 & & $<1.52$ & & ADDSCAN & K89 \\
\hline PG $1552+085$ & 0.119 & -23.3 & $<50$ & 0.80 & $<10.62$ & 22.69 & & $<1.80$ & & ADDSCAN & K89 \\
\hline PG 1612+261 & 0.131 & -23.5 & 191 & 5.07 & 11.29 & 23.57 & 0.33 & 1.58 & 1.75 & FSC & K89 \\
\hline PG $1613+658$ & 0.129 & -24.1 & 635 & 3.03 & 11.79 & 23.3 & 0.26 & 2.32 & 2.45 & S89 & K89 \\
\hline PG $1617+175$ & 0.114 & -23.6 & 102 & 1.09 & 10.89 & 23.04 & & 1.97 & & FSC & K89 \\
\hline PG $1626+554$ & 0.133 & -23.3 & $<50$ & 0.17 & $<10.72$ & 22.11 & 1 & $<2.47$ & $<2.47$ & ADDSCAN & K89 \\
\hline
\end{tabular}

${ }^{a}$ Inferred from $20 \mathrm{~cm}$ integrated emission minus flat $6 \mathrm{~cm}$ core spectrum; ${ }^{b}$ IRAS Faint Source Catalog; ${ }^{c}$ NRAO VLA Sky Survey (http://www. cv.nrao.edu/nvss/); ${ }^{d}$ own high resolution VLA measurements; ${ }^{e}$ IRAS ADDSCAN analysis; ${ }^{f}$ NASA Extragalactic Database (http://nedwww.ipac. caltech.edu).

index -0.7 for optically thin synchrotron emission, this translates to $6 \mathrm{~cm}(5 \mathrm{GHz})$ parameters in the range $1.95<u_{6}<2.75$ (mean 2.35). In fact, from radio imaging and FIR SED decomposition it is well-known that the FIR emission combines an AGN- with a star-formation component. Objects with an appreciable active nucleus display surplus radio emission, hence $u$-parameters $\lesssim 1$. In Seyfert-2 galaxy NGC 1068 for instance, roughly $80 \%$ of its $20 \mathrm{~cm}$ radio emission draws from the $1.5 \mathrm{kpc}$ triple radio source, hence is generated by the AGN. The remaining $20 \%$ occurs on larger scales, and finds its origin in host star-formation (Telesco et al. 1984). Prototypical ultraluminous starburst galaxy Arp 220 (Sanders \& Mirabel 1996) 
has $u_{6}=\log S(60 \mu \mathrm{m}) / S(5 \mathrm{GHz})=\log (103.3 \mathrm{Jy} / 0.204 \mathrm{Jy})=$ 2.70 (hence negligible AGN component), star-forming QSO Mkn 231 (Carilli et al. 1998) has $u_{6}=\log S(60 \mu \mathrm{m}) / S(5 \mathrm{GHz})=$ $\log (35.4 \mathrm{Jy} / 0.419 \mathrm{Jy})=1.93$ but $u_{6}^{\prime}=\log S(60 \mu \mathrm{m}) / S(5 \mathrm{GHz}$, extended $)=\log (35.4 / 0.173)=2.31$. In words, roughly $40 \%$ of the $5 \mathrm{GHz}$ radio emission in Mk 231 (and most of the long wavelength FIR emission) must draw from star-formation.

We therefore use sensitive, high resolution radio imaging data (references in table Col. 12) to assess - where possible - the radio-core fractions $\left(f_{\mathrm{c}}\right)$ and to transform the global, integrated $u$-parameters into AGN-subtracted $u^{\prime}$-parameters. It must be noted that we are dealing here with radio-quiet objects, i.e., objects without large scale radio lobes or jets, and that weak AGN radio emission - if any - is most likely concentrated in faint subarcsec scale radio components (cores hereafter). Inspection of the $f_{\mathrm{c}}$ entries shows several blanks in the case of objects lacking adequate radio imaging, but on the other hand very few pure-core $\left(f_{\mathrm{c}}=1\right)$ objects: diffuse galactic scale radio emission must be occurring often. Given that no cases of $f_{\mathrm{c}}>1$ are seen, the $f_{\mathrm{c}}$ values cannot be attributed to core variability combined with different observing epochs: they must reflect the presence of weak, diffuse, arcsec-scale (host galaxy scale) radio emission which is resolved out at high angular resolution. Typical diffuse luminosities are of order $10^{22}-10^{23} \mathrm{~W} / \mathrm{Hz}$, i.e., roughly one to ten times the diffuse radio emission in our Galaxy. Inspection of the table moreover shows that the extended (or integrated) radio emission frequently correlates with the integrated $60 \mu \mathrm{m}$ emission according to the radio-FIR correlation: many cases of $u_{6}$ and $u_{6}^{\prime}$ in the range 2.0-2.8 are seen. The few cases of exception (PG 0923+201, PG 0947+396, PG 1114+445, PG 1115+407, PG1435-067) display suspiciously high FIR emission which is probably due to contamination in the extended infrared beam. A few cases of mildly excessive radio emission $\left(1.5<u_{6}<2\right)$ can be also seen.

As examples we consider the QSOs PG 0052+251, PHL 909 (0054+144), and PG 1012+008. Detailed HST images of the first two objects have been presented (Bahcall et al. 1996), establishing a spiral host for the former and an elliptical host for the latter. PG $0052+251$ displays several HII regions in the disk of its host, and the HST imagery (Dunlop et al. 2003) indeed yielded a bulge/disk composite. As for PHL 909, the 2D-analysis (McLure et al. 1999) improved on the earlier (Bahcall et al. 1996) HST picture, excluding a disc host and establishing an $R=-23.62$ elliptical host having an $8 \mathrm{kpc}$ half-light radius. As seen from the table, both objects have significant diffuse host galaxy radio emission, and their AGN-corrected $u_{6}^{\prime}$-parameters fall nicely on the FIR-radio correlation. In particular, the luminous FIR emission of PHL 909 as well as the fact that $50 \%$ of its $6 \mathrm{~cm}$ radio emission must originate in extra-nuclear regions are noteworthy. While there cannot be any doubt on a disk + bulge host for PG 0051+251, also the host of PHL 909 cannot be a quiescent mature elliptical. We recall that the residual images (McLure et al. 1999; Dunlop et al. 2003) of PHL 909 display faint excess emission towards the West; additional excess emission may well be present under the QSO PSF. PG 1012+008 resides in a host galaxy which was found (Bahcall et al. 1997) to be in the process of interaction with a neighbouring galaxy. Roughly one quarter of the radio emission has a diffuse arcsec scale origin, scaling with the $60 \mu \mathrm{m}$ emission cf. the radio-FIR correlation.

In other words, the radio/FIR differences with quiescent ellipticals cannot simply be attributed to the AGN. A substantial fraction of the radio and FIR emission in these intermediate luminosity QSOs must draw from star-formation in their hosts. While in some cases this star-formation can be readily connected to residual emission in the HST imagery (after subtraction of the spheroids), in other cases it must connect to small residuals under the PSF, i.e., to star-formation within the central 100 parsecs. Given that circum-nuclear star-formation is frequently observed in Seyfert galaxies (e.g., González Delgado et al. 2001), in connection with the fact that Seyfert galaxies are generally considered to be the low-luminosity counterparts of QSOs, these results are not unexpected. We stress that we are not questioning the presence of massive bulges in the QSO hosts (and the probable connection (Dunlop et al. 2003) of the bulge/disk ratio to the QSO luminosity), but we feel urged to point out the special nature of the host ISM. Back of the envelope calculations indicate SFR's of order $10 M_{\odot}$ per year. It should be noted however that more luminous QSOs (brighter than -25) probably do not have scaled-up SFR's (Haas et al. 2003), unless at early epochs (e.g., Cox et al. 2005). Our results are nevertheless consistent with the multicolour imaging obtained by Jahnke et al. (2004a) for low redshift QSOs, as well as for more distant AGN by Jahnke et al. (2004b) and Sánchez et al. (2004). It is tempting to interpret this and other evidence for circumnuclear star-formation within the AGN accretion model of Miralda-Escudé \& Kollmeier (2005).

Finally, the above mentioned typical QSO SFR, computed from FIR data, is incompatible with the value obtained using the [OII] $\lambda 3727$ emission line strength of these QSOs. This fact - pointed out by Ho (2005) - calls for some level of extinction towards the [OII] emission, which is not unexpected given the large dust masses suggesting dusty young star clumps as f.i. also inferred in the Antennae galaxies.

\section{Summary}

We conclude that a QSO episode is not seldom accompanied or preceded by an episode of star-formation in the gas-rich circumnuclear regions of its host galaxy. We anticipate that radioloud QSOs will show the same effects, but note that radio imaging separating AGN and star-formation driven radio emission in these objects is not going to be simple. Decomposition of the FIR SEDS will be more promising in this respect: results from currently as well as soon flying infrared space observatories are eagerly awaited. The evolving dust and stellar content of QSO host galaxies will remain an issue of great importance and interest (e.g., Barthel \& Sanders 2006).

Acknowledgements. This research was initiated during a work visit to the Institute for Astronomy at the University of Hawaii; the hospitality and support of Drs. R.-P. Kudritzki and D. B. Sanders at the IfA are gratefully acknowledged.

\section{References}

Bahcall, J. N., Kirhakos, S., \& Schneider, D. P. 1996, ApJ, 457, 557 Bahcall, J. N., Kirhakos, S., Saxe, D. H., \& Schneider, D. P. 1997, ApJ, 479, 642 Barthel, P. D. 1989, ApJ, 336, 606

Barthel, P. D., \& Sanders, D. B. 2006, Proc. QSO Host Galaxies, NewAR, 50, in press

Becker, R. H., White, R. L., \& Edwards 1991, ApJS, 75, 1 (BWE91)

Brotherton, M. S., van Breugel, W., Stanford, S. A., et al. 1999, ApJ, 520, L87 Canalizo, G., \& Stockton, A. 2001, ApJ, 555, 719

Carilli, C. L., Wrobel, J. M., \& Ulvestad, J. S. 1998, AJ, 115, 928

Clements, D. L. 2000, MNRAS, 311, 833

Colina, L., \& Perez-Olea, D. E. 1995, MNRAS, 277, 845

Condon, J. J., \& Broderick, J. J. 1988, AJ, 96, 30

Cox, P., et al. 2005, in Proc. The dusty and molecular universe, ESA SP-577, 115

Dunlop, J. S., McLure, R. J., Kukula, M. J., et al. 2003, MNRAS, 340, 1095 (D03) 
Evans, A. S., Frayer, D. T., Surace, J. A. \& Sanders, D. B. 2001, AJ, 121, 1893 González Delgado, R. M., Heckman, T., \& Leitherer, C. 2001, ApJ, 546, 845 Haas, M., Klaas, U., Müller, S. A. H., et al. 2003, A\&A, 402, 87

Heckman, T. M., Smith, E. P., Baum, S. A., et al. 1986, ApJ, 311, 526

Hes, R., Barthel, P. D., \& Hoekstra, H. 1995, A\&A, 303, 8

Ho, L. C. 2005, ApJ, 629, 680

Hutchings, J. B., Crampton, D., \& Campbell, B. 1984, ApJ, 280, 41

Jahnke, K., Kuhlbrodt, B., \& Wisotzki, L. 2004a, MNRAS, 352, 399

Jahnke, K., Sánchez, S. F., Wisotzki, L., et al. 2004b, ApJ, 614, 568

Kellermann, K. I., Sramek, R., Schmidt, M., Shaffer, D. B., \& Green, R. 1989, AJ, 98, 1195 (K89)

Kauffmann, G., Heckman, T. M., Tremonti, C., et al. 2003, MNRAS, 346, 1055

Kukula, M. J., Dunlop, J. S., Hughes, D. H., \& Rawlings, S. 1998, MNRAS, 297, $366(\mathrm{Ku} 98)$

Martel, A. R., Baum, S. A., Sparks, W. B., et al. 1999, ApJS, 122, 81

McLeod, K. K., Rieke, G. H., \& Storrie-Lombardi, L. J. 1999, ApJ, 511, L67

McLure, R. J., Kukula, M. J., Dunlop, J. S., et al. 1999, MNRAS, 308, 377

Miller, P., Rawlings, S., \& Saunders, R. 1993, MNRAS, 263, 425

Miralda-Escudé, J., \& Kollmeier, J. A. 2005, ApJ, 619, 30
Rowan-Robinson, M. 1995, MNRAS, 272, 737

Sánchez, S. F., Jahnke, K., Wisotzki, L., et al. 2004, ApJ, 614, 586

Sanders, D. B , \& Mirabel, I. F. 1996, ARA\&A, 34, 749

Sanders, D. B., Phinney, E. S., Neugebauer, G., Soifer, B. T., \& Matthews, K. 1989, ApJ, 347, 29 (S89)

Schmidt, M., \& Green, R. F. 1983, ApJ, 269, 352

Scoville, N. Z., Frayer, D. T., Schinnerer, E., \& Christopher, M. 2003, ApJ, 585, L105

Tadhunter, C., Robinson, T. G., González Delgado, R. M., Wills, K., \& Morganti, R. 2005, MNRAS, 356, 480

Taylor, G. L., Dunlop, J. S., Hughes, D. H., \& Robson, E. I. 1996, MNRAS, 283, 930 (T96)

Telesco, C. M., Becklin, E. E., Wynn-Williams, C. G., \& Harper, D. A. 1984, ApJ, 282, 427

Urry, C. M., \& Padovani, P. 1995, PASP, 107, 803

Van Gorkom, J. H., Knapp, G. R., Ekers, R. D., et al. 1989, AJ, 97, 708

Walsh, D. E. P., Knapp, G. R., Wrobel, J. M., \& Kim, D.-W. 1989, ApJ, 337, 209

Wilson, A. S. 1988, A\&A, 206, 41 